\title{
Energy dissipation and recovery in a simple model with reversible cross-links
}

\author{
S. Soran Nabavi, ${ }^{1, *}$ Peter Fratzl, ${ }^{2}$ and Markus A. Hartmann ${ }^{1, \dagger}$ \\ ${ }^{1}$ Institute of Physics, Montanuniversitaet Leoben, Franz-Josef Strasse 18, 8700 Leoben, Austria \\ ${ }^{2}$ Max-Planck Institute of Colloids and Interfaces, Department of Biomaterials, Research Campus Golm, 14424 Potsdam, Germany
}

(Received 15 October 2014; published 16 March 2015)

\begin{abstract}
Reversible cross-linking is a method of enhancing the mechanical properties of polymeric materials. The inspiration for this kind of cross-linking comes from nature, which uses this strategy in a large variety of biological materials to dramatically increase their toughness. Recently, first attempts were made to transfer this principle to technological applications. In this study, Monte Carlo simulations are used to investigate the effect of the number and the topology of reversible cross-links on the mechanical performance of a simple model system. Computational cyclic loading tests are performed, and the work to fracture and the energy dissipation per cycle are determined, which both increase when the density of cross-links is increased. Furthermore, a different topology of the bonds may increase the work to fracture by a factor of more than 2 for the same density. This dependence of the mechanical properties on the topology of the bonds has important implications on the self-healing properties of such systems, because only a fast return of the system to its unloaded state after release of the load ensures that the optimal topology may form.
\end{abstract}

DOI: 10.1103/PhysRevE.91.032603

PACS number(s): 82.35.-x, 87.10.Rt, 87.15.La

\section{INTRODUCTION}

Cross-linking is a common strategy used in natural as well as in technological polymeric materials to enhance their mechanical properties [1]. One of the most prominent examples is the development of vulcanization by Goodyear in 1839 . Permanent cross-linking with sulfur bridges significantly improved the mechanical performance of rubber products. Since then many other applications for polymer cross-linking have been invented [2]. Recently covalent cross-linking was also suggested to improve the shear stability in carbon nanostructures like graphene and carbon nanotubes [3,4]. In natural materials the bending properties of actin bundles of the cell cytoskeleton differ by several orders of magnitude depending on the degree of cross-linking [5-7].

Besides permanent (covalent) cross-linking, nature also uses the concept of reversible cross-links, which provides an efficient way of toughening the material. Differently from permanent cross-links, these so-called sacrificial bonds (SBs) can open and close reversibly. SBs have been found in a large variety of biological materials such as bone $[8,9]$ and wood [10] and some fibrous materials such as silk [11,12], the mussel byssus [13-15], and the whelk egg capsule [16]. The strength of individual SBs can differ greatly, from several hundred milli-electron volts for hydrogen bridges to a value close to the strength of covalent bonds for metal coordination bonds $[17,18]$.

SBs are cross-links that are weaker than the covalent bonds that hold the structure together. Thus, upon loading, the SBs rupture first while the covalent bonds remain intact $[8,19]$.

\footnotetext{
*soran.nabavi@unileoben.ac.at

${ }^{\dagger}$ markus.hartmann@unileoben.ac.at
}

Published by the American Physical Society under the terms of the Creative Commons Attribution 3.0 License. Further distribution of this work must maintain attribution to the author(s) and the published article's title, journal citation, and DOI.
Whenever an SB fails, hidden length may be revealed by opening and unfolding of loops in the protein, and thus, energy dissipated [8]. Furthermore, SBs are often reversible; i.e., they can open and close repeatedly. Consequently, after some time the material regains its original mechanical properties when the load is released [20,21]. These remarkable properties of reversible cross-links trigger the desire to transfer some of the underlying principles to technological applications. Attempts include the development of self-healing polymeric materials with increased stiffness and extensibility [22-24], mechanical improvement of hydrogels [25-28], fabrication of mussel-inspired polydopamine films for use in biomedical or electrochemical applications [29-31], and functionalization of graphene using mussel-inspired chemistry [32]. Similar concepts have been used to reinforce weak elastomers with stiff fillers. In these concepts, the bonds between the elastomer and the fillers act as SBs [33]. Upon cyclic loading filled elastomers often show a pronounced hysteresis and a deformation-induced softening during the second loading cycle, the so-called Mullins effect. This softening arises because the bonds between elastomer and filler that re-form during unloading are reduced in strength and stiffness compared to the original bonds [34]. Theoretical approaches to describing this effect range from macroscopic continuum models using constitutive equations [35-39] to microscopic models using molecular dynamics simulations [40].

Understanding the underlying structure-function relationships that allow for this extraordinary mechanical performance is of utmost importance to mimic these fundamental design principles and to transfer them into novel man-made materials [41]. This understanding, nevertheless, is an extremely complicated task due to the enormous complexity and subtle interactions that are found in biological systems. Thus, simple models focusing on well-defined aspects of the problem may be addressed either analytically or with the help of computer simulations and are indispensable tools making these complex systems theoretically tractable and helping the interpretation of experimental findings. Using analytical and computational modeling techniques, it was shown that confinement strongly 
influences the rupture of hydrogen bonds in silk-like structures $[42,43]$, that the distribution of SBs determines the shear deformability in bone [44], and that mechanical properties of structures relying on SBs show a pronounced dependence on pulling speed $[45,46]$. Furthermore, it should be noted that the role of $\mathrm{SBs}$ in the mechanical properties of the investigated system is completely analogous to the role of reversible cross-links in other polymeric systems, e.g., actin fiber bundles $[47,48]$.

In the present paper we investigate a simple model of a single-polymer chain that can reversibly form cross-links between some of its monomers mimicking the effect of SBs. Using Monte Carlo (MC) simulations we investigate the influence of SB density and topology on the work to fracture and specifically on the amount of dissipated energy per loading cycle. Special emphasis in the discussion is put on the process of re-forming ruptured SBs during unloading of the system. This re-forming determines the mechanical properties after one loading cycle and is thus strongly linked to the self healing capabilities of the structures. The mechanical properties depend on the topology that SBs form when closing. The type of topology is strongly influenced by the rate at which the loaded structure returns into its native state. The approach chosen in this paper is to build an especially simple model, capturing the essentials of sacrificial bonding. While the model is too simple and generic to allow modeling of any special experimental system quantitatively, it provides the advantage that its thermodynamic and mechanical behavior can be understood completely. As a next step the basic mechanisms underlying energy dissipation and recovery identified in this model will also help to understand the much more complex situation in real systems.

\section{THE MODEL}

The model is inspired by the fascinating class of metalloproteins and was already presented in [49]. Thus, in the following only a short description is given focusing on the new concepts used in this paper. This work is inspired by proteins, where the segments which carry SBs are relatively short with only 30 to 80 amino acids [68]. Consequently, our model consists of a single, linear chain of $N=50$ covalently bonded beads with a hard-sphere radius $R$ (that we set as the unit of length). Thus, one bead in the model roughly corresponds to one amino acid. The energetics of the covalent bonds are described via a Morse potential as shown is Fig. 1:

$$
E\left(r_{i j}\right)=E_{0}\left\{\left[1-\exp \left(-\beta\left(r_{i j}-r_{0}\right)\right)\right]^{2}-1\right\}
$$

$E_{0}=5 \mathrm{eV}$ is the depth of the potential, $\beta^{-1}=0.5 \mathrm{R}$ the width of the potential, $r_{0}=3 R$ the equilibrium distance and $r_{i j}$ is the distance between the two neighboring beads. Consequently the contour length of the chain is given by $L_{C}=$ $(N-1) r_{0}$. To account for the effect of reversible cross-links $N_{s}$ of the beads are defined as sticky. The sticky sites are introduced regularly, i.e., the same number of non-sticky sites separating them. Always two of these sticky sites could form a SB. The energetics of the SBs are described with an identical potential as the covalent bonds but with a reduced binding energy. Because experimental findings indicate that a typical metal coordination bond has a strength of approximately $20 \%$ (a)
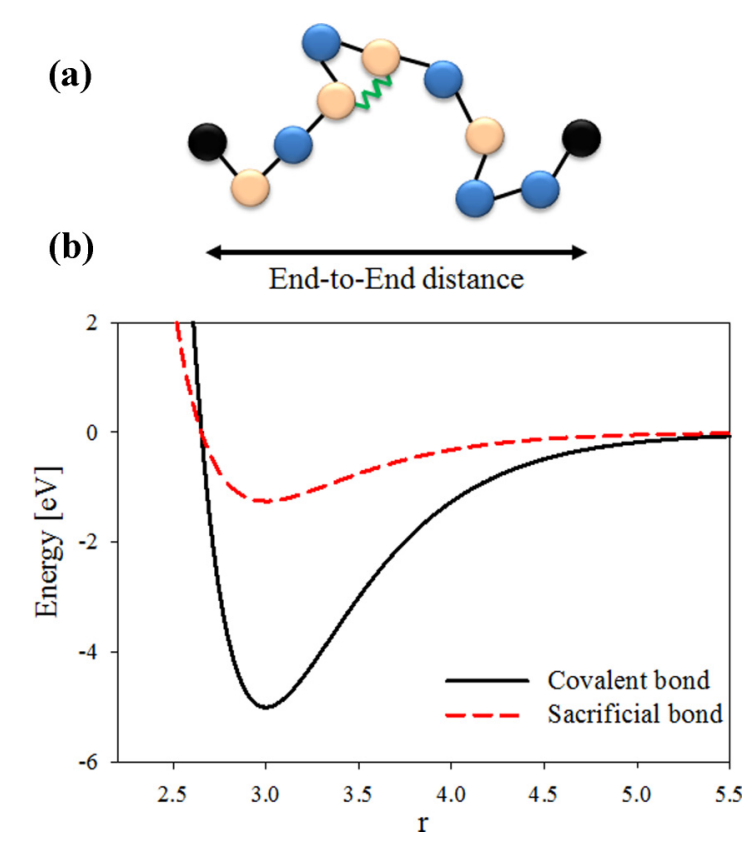

FIG. 1. (Color online) (a) Schematic of the model. Black spheres denote the two fixed outer beads defining the end-to-end distance $L$; dark and light spheres correspond to nonsticky and sticky sites, respectively. Covalent bonds are indicated by black lines, while a closed sacrificial bond is shown by the zigzag (green) line. (b) Morse potentials of different binding energies are used to describe the energetics of covalent and sacrificial bonds as shown by the solid and dashed curves, respectively.

to $30 \%$ of a covalent bond $[17,60]$ we set $E_{0}^{\mathrm{SB}}=1.25 \mathrm{eV}$ (see Figs. 1(a) and 1(b)). The cross-links were allowed to open and close reversibly and cross-link updating was performed using a standard Metropolis algorithm [50]. Updating the position of the inner beads of the chain was also performed using the Metropolis algorithm. Simulations were performed in the Helmholtz ensemble by pinning the first and last bead defining the end-to-end distance $L=\left|r_{1}-r_{N}\right|$ of the chain [51]. Simulations mimicking cyclic loading experiments were performed by starting from a small end-to-end distance that was gradually increased until $L / L_{C}=1$. Then the loading was reversed, i.e. $L$ was slowly decreased, until the initial end-to-end distance was recovered. Eventually the chain was then stretched a second time. During each simulation step the force on the outer beads was recorded and averaged. For each length up to 3 million MC steps, i.e., jump trials per bead and per sticky site, were performed. The results reported in this paper are the averages of 20 independent runs for stretching and 10 independent runs for unloading of the chain. In the simulations the temperature was set to the ambient value of $k_{B} T=25 \mathrm{meV}$.

\section{RESULTS}

\section{A. Influence of the sticky site density}

Independent starting configurations with small end-to-end distances were prepared by slowly unloading a fully stretched chain without sticky sites to the starting length $L / L_{C}=$ 0.04. Then the sticky sites were introduced corresponding 


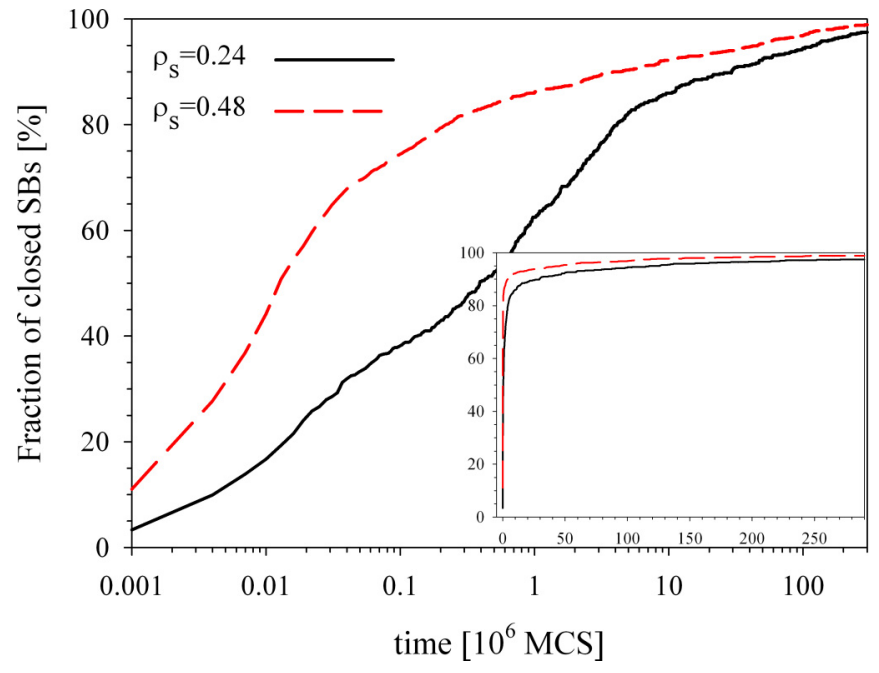

FIG. 2. (Color online) Number of closed SBs as a function of time for the two high SB densities. Data are the average of 100 independent runs for each sticky site density. Note the logarithmic scaling of the time axis. Inset: The same data with a linear time axis.

to densities $\rho_{s}=N_{s} / N=0.08,0.24$, and 0.48 , respectively, and SBs were allowed to form. Figure 2 shows the average number of closed SBs as a function of the time measured in units of MC steps and the two densities of sticky sites. At both densities there is an initial steep increase in sticky site number that subsequently slows down at longer times. This behavior is similar to what was shown in experiments that report an initially fast recovery of the mechanical properties, reaching approximately $70 \%$ after waiting times of $1 \mathrm{~h}$ but slowing down considerably, to a recovery of only $95 \%$, after $168 \mathrm{~h}[13]$.

Figure 3 shows load-displacement curves for cyclic loading and the three investigated sticky site densities obtained after the SBs had formed. Black symbols show the behavior during loading, while gray (red) symbols denote unloading of the structure. The bottom row in the same figure shows the corresponding number of intact SBs. The lowest density corresponds to $N_{s}=4$ sticky sites in the system, which are separated by 10 monomers. When this structure is stretched a discrete rupture of SBs can be observed [see Fig. 3(a)]. The position of the observed force peaks is determined by the topology of the involved SBs, while its height is intimately tied to the thermal fluctuations of the protein backbone and is considerably smaller than the theoretical strength of one $\mathrm{SB}$, given by $F_{\max }=\beta E_{0}^{\mathrm{SB}} / 2=1.25 \mathrm{eV} / \mathrm{R}$ [49]. Each single peak can be fitted with the worm-like-chain model [1,52], which has been shown to describe such entropic loads reasonably well $[53,54]$. When $\rho_{s}$ is increased (corresponding to $N_{s}=12$ and 24 sticky sites, respectively) the distinct peaks corresponding to SB rupture merge into one large plateau and the force does not drop to 0 between discrete rupture events [see Figs. 3(c) and 3(e)]. The height of this plateau is comparable to the height of the distinct force peaks shown in Fig. 3(a); in particular, the plateau is also lower than the theoretical strength of one SB. Thus, entropic effects continue to largely influence the effective strength of SBs also for high sticky site densities.

As soon as the polymer is stretched to its contour length $L_{C}$, the direction of loading is reversed and the structures are unloaded. For all three sticky site densities the load-displacement curves show a pronounced asymmetry between loading and unloading: more energy is needed to elongate the polymer

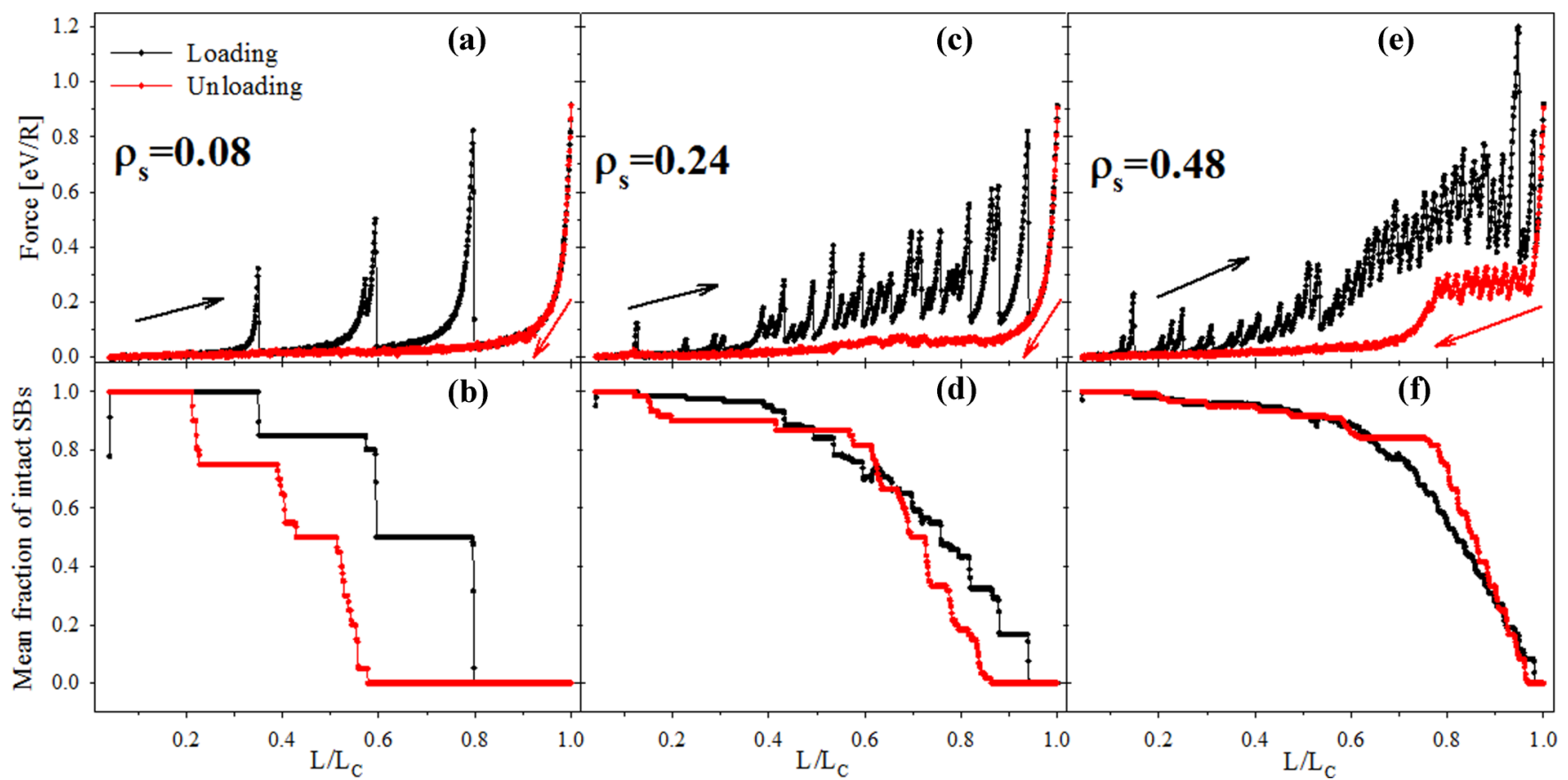

FIG. 3. (Color online) (a), (c), (e) Cyclic loading curves for $N=50$ at $k_{B} T=25 \mathrm{meV}$ and three sticky site densities. (b), (d), (f) Corresponding mean number of closed SBs as a function of the end-to-end distance. Black symbols denote loading branches; gray (red) symbols, unloading branches. Curves are the averages of 20 runs of stretching and 10 runs of unloading. 
TABLE I. The energy needed to stretch the molecule to its contour length $E_{1}$ (up to an additive constant this corresponds to the work to fracture), the energy gained during unloading $E_{2}$, the corresponding energy dissipation $\Delta E=E_{1}-E_{2}$, and the amount of dissipated energy $\Delta E / E_{1}$ for the different investigated structures.

\begin{tabular}{|c|c|c|c|c|}
\hline & $\begin{array}{c}E_{1} \\
(\mathrm{eV})\end{array}$ & $\begin{array}{c}E_{2} \\
(\mathrm{eV})\end{array}$ & $\begin{array}{c}\Delta E \\
(\mathrm{eV})\end{array}$ & $\Delta E / E_{1}$ \\
\hline \multicolumn{5}{|c|}{ Crumpled starting configuration } \\
\hline$\rho_{s}=0.08$ & $11.4 \pm 0.2$ & $5.90 \pm 0.02$ & $5.5 \pm 0.2$ & $0.48 \pm 0.02$ \\
\hline$\rho_{s}=0.24$ & $22.6 \pm 0.5$ & $7.42 \pm 0.06$ & $15.1 \pm 0.5$ & $0.67 \pm 0.03$ \\
\hline$\rho_{s}=0.48$ & $36.7 \pm 1.3$ & $12.84 \pm 0.14$ & $23.9 \pm 1.3$ & $0.65 \pm 0.04$ \\
\hline \multicolumn{5}{|c|}{ Predefined topologies } \\
\hline Independent & $24.2 \pm 0.1$ & $12.84 \pm 0.14$ & $11.3 \pm 0.2$ & $0.47 \pm 0.01$ \\
\hline Nested & $54.6 \pm 0.5$ & $12.84 \pm 0.14$ & $41.7 \pm 0.6$ & $0.76 \pm 0.01$ \\
\hline Pseudoknotted & $63.0 \pm 0.7$ & $12.84 \pm 0.14$ & $50.1 \pm 0.7$ & $0.80 \pm 0.01$ \\
\hline \multicolumn{5}{|c|}{ Second stretching } \\
\hline & $30.0 \pm 1.2$ & $12.84 \pm 0.14$ & $17.2 \pm 1.2$ & $0.57 \pm 0.05$ \\
\hline
\end{tabular}

than is restored when it is unloaded. The difference in the area of the two curves is a measure of the dissipated energy per loading cycle $\Delta E=E_{1}-E_{2}$, with $E_{1}$ and $E_{2}$ the area under the loading and the unloading curve, respectively. The work to fracture is given by $W=E_{1}+E_{C}$, with $E_{C}=25 \mathrm{eV}$ the energy needed to finally rupture the fully elongated polymer. Because all SBs are open for a fully elongated polymer, $E_{C}$ is a constant for all investigated structures. Thus, in the following, $E_{C}$ is omitted and the energy to elongate the polymer $E_{1}$ and the work to fracture are used interchangeably. For the three investigated densities the dissipated energy was found to be $\Delta E=5.5 \pm 0.2,15.1 \pm 0.5$, and $23.8 \pm 1.4 \mathrm{eV}$, respectively. The corresponding relative amount of dissipated energy was given by $\Delta E / E_{1}=0.49,0.67$, and 0.65 , respectively (see also Table I). Figures 3(b), 3(d), and 3(f) show that all SBs are open when the chains are stretched to their contour lengths. Upon unloading of the chain with $\rho_{s}=0.08$ the SBs re-form, but at end-to-end distances smaller than the distance corresponding to bond breaking [see Fig. 3(b)]. At these smaller distances the chain already has enough conformational freedom that the formed SBs can relax and, thus, do not experience an elevated force. To understand the asymmetry between loading and unloading, think of a simple system with two sticky sites only. At the start of the loading cycle at a small end-to-end distance the SB is closed. This bond fails at an end-to-end distance $L^{*}$ when the free length of the chain is almost completely stretched. When the chain is approaching $L^{*}$ from above during unloading the SB is open, and thus, the monomers are free to fluctuate. However, formation of a stable SB requires that the two sticky sites come into close spatial proximity. At $L^{*}$ this is only possible if the free length of the chain is almost completely stretched, as was enforced during loading by the closed SB. It is highly improbable for the chain taking this stretched configuration randomly, because of the low number of corresponding configurations, i.e., the low entropy of this state.

Unloading of the chain with $\rho_{s}=0.24$ is similar to the low-density case $\rho_{s}=0.08$. Mostly, the bonds re-form at distances shorter than when they were broken. At higher sticky site densities the distance between neighboring sites is smaller and the force is slightly increased compared to unloading for the case $\rho_{s}=0.08$ (see Fig. 3). The small region around
$L / L_{C}=0.6$, where sticky sites re-form earlier than they were broken, is due to SBs that were originally formed between sticky sites separated by a long distance along the chain that re-form between closer sticky sites.

This effect is even more pronounced for the highest sticky site density, $\rho_{s}=0.48$, whose unloading behavior can be described as a three-stage process [Figs. 3(e) and 3(f)]: First, for an end-to-end distance still close to the contour length the number of intact SBs increases considerably due to bond formation between sticky sites that are in close vicinity along the chain. This different behavior compared to the low-density case is explained by (i) the higher number of sticky sites and (ii) their closer distance along the chain, which both increase the probability of SB formation. In contrast to the low-density case, due to the increased length of the chain these bonds do not yet have the conformational freedom to relax. Thus, the SBs are still strained, resulting in the plateau in the unloading part of the load-displacement curve. Second, for intermediate end-to-end distances no new bonds are formed, but the existing bonds relax. This can be seen as a sharp drop in the force in the load-displacement curve [Fig. 3(e)] and a constant number of intact bonds [Fig. 3(f)]. Third, for small end-to-end distances the chain is relaxed enough that the remaining sticky sites that are far apart along the chain can form SBs. Thus, the number of intact SBs slowly increases again, while the force is slowly decaying to 0 . For low $\rho_{s}$ all sticky sites are well separated along the chain and unloading of the chain is described by the third stage only [see Fig. 3(a)].

The equilibration times in the simulations were chosen to be long enough that the system was in equilibrium at each elongation. Thus, the observed hysteresis loops for all densities are static in nature, meaning that the hysteresis area does not vanish when the frequency of cycling tends to 0 . Such behavior is linked to bistable systems. In the system presented the two states consist of different (stable) states of SBs at the same elongation (open or closed and formed between different partners, respectively). We believe that this behavior is different from collective first-order phase transitions that have been reported, e.g., for the unbinding transition of filament bundles upon a decrease in the linker concentration [55] or force-induced desorption or stiffening of fibers $[48,56]$. 
(a): Independent

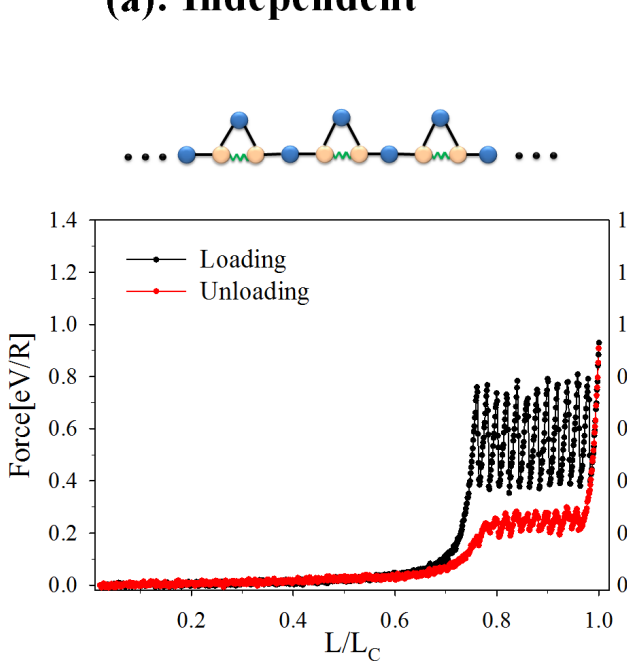

(b):Nested

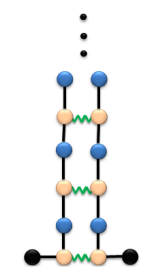

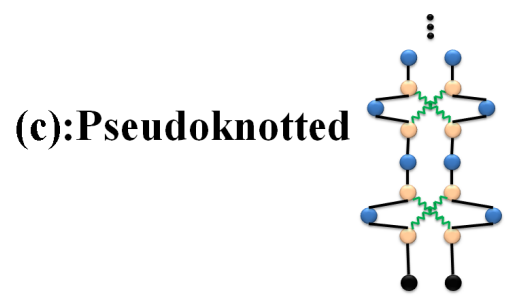
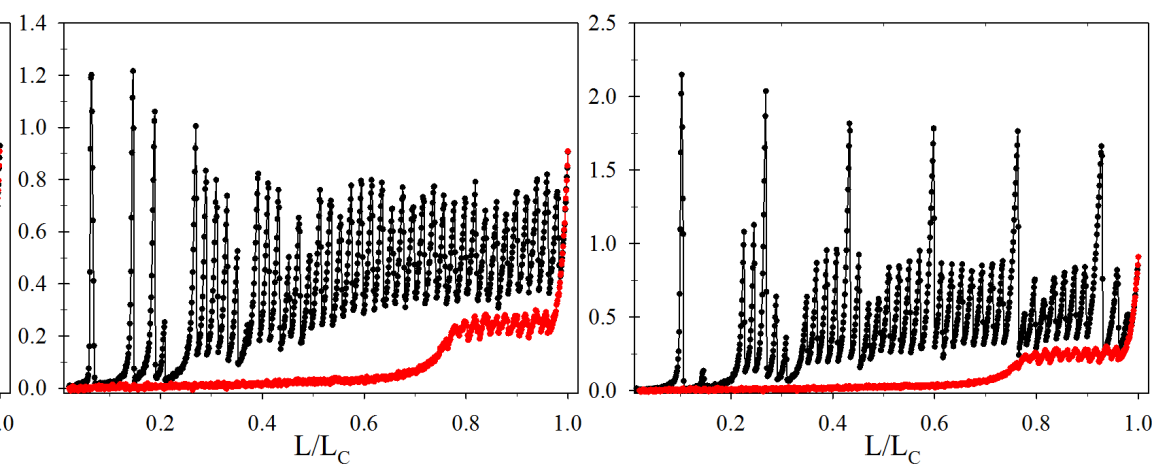

FIG. 4. (Color online) Starting configurations (top) and load-displacement curves (bottom) for three topologies of SBs: independent (a), nested (b), and pseudoknotted (c). Black spheres denote fixed end beads; dark and light beads denote nonsticky and sticky sites, respectively. Straight connections correspond to the covalent backbone of the structure; zigzag connections denote closed SBs. Note that in the independent configuration SBs are formed between neighboring sticky sites, while the other two topologies are characterized by SBs connecting sticky sites that are far apart in the chain. In the load-displacement curves the black line shows stretching of the different starting configurations until the contour length, and the gray (red) line shows subsequent (quasistatic) unloading. Note that this curve is identical for all structures. The length of the chains was $N=50$, and the sticky site density $\rho_{s}=0.48$.

\section{B. Influence of the topology of bonds}

Previous work has shown that the topology of bonds has a large influence on the mechanical behavior of the polymer and its capability of dissipating energy [49,57-59]. To test the influence of the topology on $E_{1}$ and the dissipated energy $\Delta E$ a new set of simulations with special starting configurations was performed. Instead of introducing the sticky sites in the crumpled starting state of the polymer and allowing the formation of SBs without any bias, in these new starting configurations the topology of the SBs was predefined at the beginning: independent, nested, and pseudoknotted configurations (see the top row of Fig. 4 for a sketch of the starting configurations and corresponding topologies) [57]. The bottom row in Fig. 4 shows the resulting load-displacement curves. Black symbols denote loading of the different starting configurations. Although the number of sticky sites and thus the initial number of SBs are equal for all three structures $\left(\rho_{s}=0.48\right)$, the stretching part of the load-displacement curves shows a considerably different behavior. For the independent configuration the force starts rising at the largest elongations compared to the other two topologies. The first SB starts stretching when the length of the structure is increased to $L / L_{C}=1-N_{s} / 2(N-1) \approx$ $1-\rho_{s} / 2=0.76$ [see Fig. 4(a)], while much lower values, $L / L_{C} \approx 3 /(N-1)=0.06$ and $L / L_{C} \approx 5 /(N-1)=0.10$, are found for the nested and the pseudoknotted configurations, respectively. Another difference between the topologies can be seen in the number of single rupture events characterized by the force peaks in the load-displacement curves. There are exactly 12 rupture events for the independent configuration, while for the nested and pseudoknotted configurations the number of rupture events increases by a factor of more than 3. In all the configurations there are 24 sticky sites, giving a maximum number of 12 simultaneously closed SBs. The number of 12 SB ruptures for the independent configuration shows that after SB rupture the now open sticky sites do not re-form. This is different for the nested and pseudoknotted configurations. The number of SB ruptures is much larger than 12, indicating that in this case after rupture the open sticky sites recombine, leading to the formation of new SBs. It is clear that the re-forming of SBs increases the work under the stretching curve. On the other hand, the lack of SB re-formation leads to a reduction of more than $50 \%$ in $E_{1}$ for the independent topology compared to the other two cases (see Table I). Another point that can be observed concerns the height of the force peaks corresponding to individual SB rupture events. Although in the simulations all SBs are identical, the height of the observed force peaks shows significant variations. In Ref. [49] it was shown that the effective strength of SBs is reduced by thermal backbone fluctuations and, thus, crucially depends on the effective length of the chain defined by the SB. This effect is clearly shown in Fig. 4. For the independent configuration SB rupture starts at the large effective length of $L / L_{C} \approx 0.75$ and all the force peaks show a height of $\approx 0.8 \mathrm{eV} / \mathrm{R}$, which is a reduction of one-third compared to the theoretical strength of one SB of $F_{\max }=1.25 \mathrm{eV} / \mathrm{R}$ [see Fig. 4(a)]. The first rupture in the nested configuration occurs at the small effective length of $L / L_{C} \approx 0.06$. Due to this small length the effective strength of the SB is close to its theoretical value $F_{\max }$. When the chain is further stretched its effective length increases and the 
effective strength of SBs decreases, attaining the same value, $\approx 0.8 \mathrm{eV} / \mathrm{R}$, as in the independent configuration for large elongations [see Fig. 4(b)]. The rupture of the six pseudoknotted loops in Fig. 4(c) results in force peaks approximately twice the size of the single SBs. Also, here the reduction of the effective strength with increasing elongation is clearly visible.

When the chains were stretched to $L / L_{C}=1$ the loading was reversed and the chains unloaded. At this point all SBs were open. Thus, the starting configuration, and consequently the behavior upon unloading, is the same for all cases with the same sticky site density [especially, it is also identical to the unloading already discussed and shown in Fig. 3(e)].

\section{DISCUSSION}

The simple model presented here cannot claim to describe the microscopic details of enormous complexity characterizing load-bearing structures in real materials. The polymer itself is described by a simple bead model, the backbone elasticity is given solely by bond stretching contributions, all other monomer interactions are of hard sphere type only (excluded volume interactions), and the solvent-and consequently the solvation entropy-is totally neglected. Furthermore, in contrast to molecular dynamics or Brownian dynamics simulations, the MC method evaluates time-independent, static properties. Thus, vibrational entropy is neglected. SBs are described phenomenologically with an effective potential connecting always two beads, whereas in natural and technological materials SBs are often formed among three partners [14]. Despite these massive simplifications, which are necessary to make the situation computationally tractable, many characteristics found in the mechanical behavior of biological materials can also be found in the model presented. The sawtooth pattern characteristic of single SB rupture for low sticky site densities [see Fig. 3(a)] closely resembles experimentally found loading curves reported in nacre [19] or in single-molecule measurements [60-65]. Furthermore, experimental investigations of the mussel byssus show an energy dissipation of $\Delta E / E_{1} \approx 0.7$ [66], which is similar to the values found during cyclic loading for the two high sticky site densities investigated in this paper.

One of the most remarkable properties of biological materials is their ability to repair and self-heal. One concept of nature that achieves this is the use of reversible SBs. It is the reversibility of these bonds that allows for repair after the load is released. Nevertheless, the repair takes some time and healing does not occur instantaneously. Whenever the structure is reloaded immediately after unloading the mechanical properties characterizing the second stretching are strongly deteriorated compared to the first, while the mechanical properties recover after some waiting time between consecutive loading cycles [67]. This effect is attributed to the time needed for the sticky sites (which are mostly open after loading) to find and re-form stable SBs. Experiments showed that this process speeds up when the temperature is increased, thus indicating that SB formation is a random, thermally activated process [13]. The rate of bond formation obtained with the present model resembles the experimental finding of an initially fast formation that subsequently slows

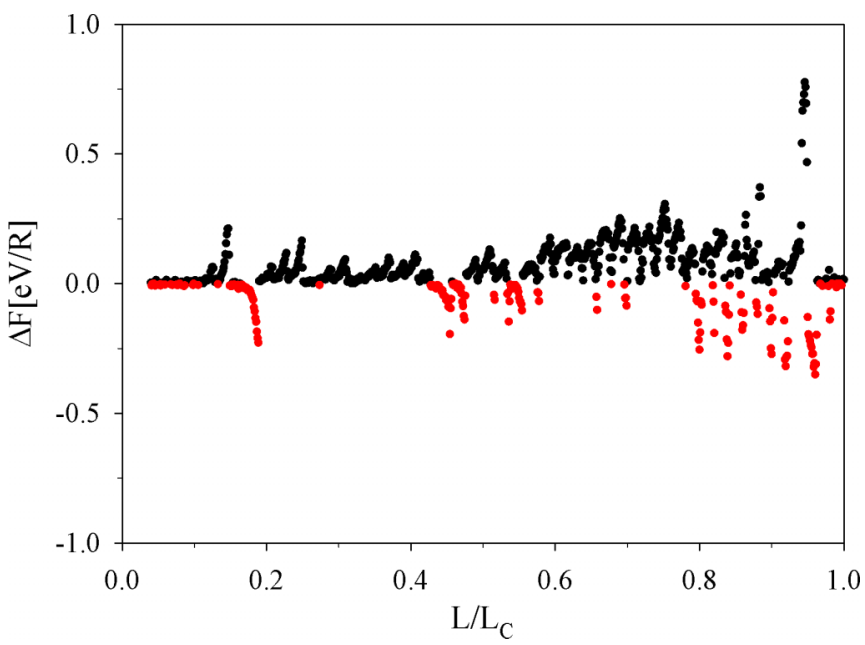

FIG. 5. (Color online) Difference $\Delta F=F_{1}-F_{2}$ of the loaddisplacement curves for the first vs second stretching. Black circles denote $\Delta F>0$; gray (red) circles, $\Delta F<0$. Clearly, on average $\Delta F>0$, showing that the first stretching cycle shows on average a higher load than the second. This results in an approximately $20 \%$ decrease in $E_{s}$; see also Table I.

down (see Fig. 2), further indicating that the recovery is a stochastic, thermally activated process. Nevertheless, one should keep in mind that the self-healing process in reality is much more complicated than can be captured with the simple model presented, depending, e.g., on the $\mathrm{pH}[68,69]$ and the $\mathrm{p} I$ value [70].

Not only the re-formation of SBs but also all relevant mechanical parameters depend on time, i.e., stretching speed [46,71]. Energy dissipation becomes greater if the structure is unloaded more rapidly than the SBs can form, i.e., $\Delta t_{\text {Unload }} \ll \tau_{\mathrm{SB}}$. No elevated force would be seen on the unloading branch, effectively increasing the area between the curves. On the other hand, energy dissipation would decrease when stretching is faster than the relaxation time of the polymer, such that it is not the SBs but rather the covalent bonds that fail. Although MC simulations do not allow us to directly determine time-dependent properties, it is still possible to gain some insights into the two limiting cases of infinitely fast and infinitely slow (quasistatic) (un)loading. The load-displacement curves presented in Figs. 3 and 4 correspond to quasistatic deformation i.e., the polymer was stretched so slowly that the structure was given enough time to equilibrate at each loading step. When these quasistatically unloaded structures are stretched a second time, this second stretching is different from the first. This is due to the different preparation of the starting state. The starting configuration for the first stretching was prepared such that SB formation was allowed only when the polymer was fully unloaded. This corresponds to unloading that is much faster than the SBs can form, i.e., the limiting case of infinitely fast unloading $\Delta t_{\text {Unload }} \ll \tau_{\mathrm{SB}}$. In contrast, the starting configuration for the second stretching was obtained by a quasistatic unloading of the polymer, allowing for SB formation already at large elongations. Figure 5 shows the difference in the first versus second stretching cycle, $\Delta F=F_{1}-F_{2}$, calculated from the averaged load-displacement curves as a function of elongation. 
Clearly, on average, $\Delta F>0$, showing that the load of the second stretching is reduced compared to the first. This leads to a $20 \%$ reduction in the area under the stretching curve from 36.7 to $30 \mathrm{eV}$ (see Table I). The different mechanical behavior of the second stretching cycle can be attributed to the changed topology of the SBs induced by the different methods of preparation of the starting configuration. On average, $72 \pm$ $3.4 \%$ independent, $14.5 \pm 2.4 \%$ nested, and $13.5 \pm 2.1 \%$ pseudoknotted SBs are formed for the fast unloading. In contrast, quasistatic unloading favors the independent topology (SBs are formed between neighboring sticky sites), at the cost of the pseudoknotted structure (SBs are formed between non-neighboring sticky sites). A detailed analysis showed that during quasistatic unloading $83 \pm 3 \%$ of the SBs show the independent topology. The remaining $17 \pm 3 \%$ of SBs are of the nested type, while not a single pseudoknotted configuration was created in any unloading simulation run. It is this decrease in the number of pseudoknotted SBs that is responsible for the roughly $20 \%$ decrease in $E_{1}$ for the second stretching cycle after a quasistatic return. Thus, in the investigated model a fast unloading is essential to achieve a complete recovery of the initial material properties, i.e., the unloading has to be much faster than the time scale of SB re-formation. A similar mechanism might explain why the mussel byssus possesses a highly ordered elastic framework that ensure a very fast return of the stretched structure upon release of the load [72]. An analogous situation is found for cyclic loading of titin, where the second stretching cycles are characterized by a lower number of the characteristic sawtooth peaks compared to the first [61].

As discussed above for random polymers a fast return of the structure into its unstrained state can give an approximately $20 \%$ increase in the work to fracture. This increase can be enhanced to almost $100 \%$ if it is possible to favor the nested or pseudoknotted over the independent topology (see Table I). This can be achieved if the polymer shows a certain folding pattern. While random polymers have to rely on thermally induced formation of SBs governed by the fluctuations of the backbone, the advantage of folding is that it is possible to bring selected sticky sites into close spatial proximity so that they can form SBs of a chosen topology. A single polymer chain with SBs in the nested or pseudoknotted configuration requires more than double the amount of energy to be elongated compared to the independent configuration. While proteins control their folding pattern via the mutual interaction of the sidechains and of the sidechains with water, one of the most important factors determining the shape of polymers in general is the backbone elasticity. When additional bending terms are included in the description of the backbone elasticity of the polymers, depending on the magnitude of the bending constant and the temperature, a broad range of equilibrium shapes of the polymers, including hairpin structures, can be observed [73]. Naturally these hairpin structures favor the formation of the nested topology. Another example are SBs based on hydrogen bridges in $\beta$ sheets that are responsible for the remarkable mechanical properties of silk $[42,43]$. The parallel strands allow for the pseudoknotted topology. The resulting cooperative failure of the SBs maximizes the force to unfold the polymer [71,74]. Furthermore, due to the cooperative loading of the SBs in these structures the rather weak hydrogen bridges can provide considerable forces. On the other hand, to provide sufficient mechanical stability SBs in random coil polymers like the metal coordination bonds investigated in this paper are much stronger, because they normally fail sequentially.

\section{SUMMARY AND CONCLUSIONS}

Using a simple model the influence of the number and topology of SBs on the mechanical behavior of polymeric chains during cyclic loading was investigated. In general both the work to fracture $E_{1}$ and the energy dissipation $\Delta E$ increase with increasing sticky site density. While for low sticky site densities a discrete rupture of single SBs can be observed, for high sticky site densities these single peaks merge into one large plateau. Computational cyclic loading experiments showed a pronounced asymmetry between the stretching and the unloading branch for all sticky site densities. This hysteresis is due to two reasons: first, the SBs re-form at elongations smaller than those at which they rupture; and second, the SBs re-form between sticky sites separated by smaller distances along the chain than when the SBs originally formed. Consequently, upon quasistatic unloading the topology of the SBs changes. In general, the amount of SBs of the independent type increases at the cost of the pseudoknotted type.

It was shown that the nested and pseudoknotted topologies possess superior mechanical properties compared to the independent configuration. The work to fracture and the dissipated energy are increased by a factor of more than 2 from the purely independent to the pseudoknotted topology. This large increase is due to a pronounced re-forming of the bonds ruptured during loading. After a quasistatic unloading the formation of SBs of the independent type are favored, at the cost of the pseudoknotted behavior. Thus, the mechanical performance of the polymer deteriorates when it is stretched a second time. It can be concluded that after release of the load a fast return to its initial state is essential for the polymer to maintain its mechanical performance.

Although simple, the model presented captures several aspects of sacrificial bonding also found in real systems. These are, first, the characteristic sawtooth patterns found for low sticky site densities; second, the pronounced hysteresis in cyclic loading with an energy dissipation of $\approx 70 \%$ for high sticky site densities; third, the characteristic time scale of re-formation of SBs after unloading, with an initial fast and a subsequent slow recovery; and fourth, the deterioration of mechanical properties during second loading, depending on the unloading speed. This resemblance might indicate that the mechanisms of energy dissipation and recovery identified in this simple model also have some importance in real systems.

\section{ACKNOWLEDGMENTS}

We thank M. J. Harrington for fruitful discussions. S.N. and M.H. gratefully acknowledge financial support from the Austrian Science Fund (FWF) in the framework of project $\mathrm{P}$ 22983-N20. 
[1] M. Rubinstein and R. H. Colby, Polymer Physics (Oxford University Press, New York, 2003).

[2] A. Abe, K. Dusek, and S. Kobayashi (eds.), Crosslinking in Materials Science. Advances in Polymer Science (Springer, Berlin, 2005), Vol. 184.

[3] R. H. Telling, C. P. Ewels, A. A. El-Barbary, and M. I. Heggie, Nat. Mater. 2, 333 (2003).

[4] A. Kis, G. Csányi, J.-P. Salvetat, T.-N. Lee, E. Couteau, A. J. Kulik, W. Benoit, J. Brugger, and L. Forró, Nat. Mater. 3, 153 (2004).

[5] M. M. A. E. Claessens, M. Bathe, E. Frey, and A. R. Bausch, Nat. Mater. 5, 748 (2006).

[6] S. Ulrich, A. Zippelius, and P. Benetatos, Phys. Rev. E 81, 021802 (2010).

[7] P. Benetatos, S. Ulrich, and A. Zippelius, New J. Phys. 14, 115011 (2012).

[8] G. E. Fantner, T. Hassenkam, J. H. Kindt, J. C. Weaver, H. Birkedal, L. Pechenik, J. A. Cutroni, G. A. G. Cidade, G. D. Stucky, D. E. Morse et al., Nat. Mater. 4, 612 (2005).

[9] H. S. Gupta, P. Fratzl, M. Kerschnitzki, G. Benecke, W. Wagermaier, and H. O. K. Kirchner, J. R. Soc. Interface 4, 277 (2007).

[10] J. Keckes, I. Burgert, K. Frühmann, M. Müller, K. Kölln, M. Hamilton, M. Burghammer, S. V. Roth, S. Stanzl-Tschegg, and P. Fratzl, Nat. Mater. 2, 810 (2003).

[11] E. Oroudjev, J. Soares, S. Arcidiacono, J. B. Thompson, S. A. Fossey, and H. G. Hansma, Proc. Natl. Acad. Sci. USA 99, 6460 (2002).

[12] N. Becker, E. Oroudjev, S. Mutz, J. P. Cleveland, P. K. Hansma, C. Y. Hayashi, D. E. Makarov, and H. G. Hansma, Nat. Mater. 2, 278 (2003).

[13] M. J. Harrington, H. S. Gupta, P. Fratzl, and J. H. Waite, J. Struct. Biol. 167, 47 (2009).

[14] M. J. Harrington, A. Masic, N. Holten-Andersen, J. H. Waite, and P. Fratzl, Science 328, 216 (2010).

[15] D. S. Hwang, H. Zeng, A. Masic, M. J. Harrington, J. N. Israelachvili, and J. H. Waite, J. Biol. Chem. 285, 25850 (2010).

[16] M. J. Harrington, S. S. Wasko, A. Masic, F. D. Fischer, H. S. Gupta, and P. Fratzl, J. R. Soc. Interface 9, 2911 (2012).

[17] D. S. Barnes and L. D. Pettit, J. Inorg. Nucl. Chem. 33, 2177 (1971).

[18] Z. Xu, Sci. Rep. 3, 2914 (2013).

[19] B. L. Smith, T. E. Schäffer, M. Viani, J. B. Thompson, N. A. Frederick, J. Kindt, A. Belcher, G. D. Stucky, D. E. Morse, and P. K. Hansma, Nature 399, 761 (1999).

[20] W. C. Yount, D. M. Loveless, and S. L. Craig, Angew. Chem. Int. Ed. 44, 2746 (2005).

[21] L. Schmitt, M. Ludwig, H. E. Gaub, and R. Tampe, Biophys. J. 78, 3275 (2000).

[22] A. M. Kushner, V. Gabuchian, E. G. Johnson, and Z. Guan, J. Am. Chem. Soc. 129, 14110 (2007).

[23] P. Cordier, F. Tournilhac, C. Soulié-Ziakovic, and L. Leibler, Nature 451, 977 (2008).

[24] Y. Yang and M. W. Urban, Chem. Soc. Rev. 42, 7446 (2013).

[25] M. A. Haque, T. Kurokawa, G. Kamita, and J. P. Gong, Macromolecules 44, 8916 (2011).

[26] M. A. Haque, T. Kurokawa, and J. P. Gong, Polymer 53, 1805 (2012).
[27] J. Hu, T. Kurokawa, T. Nakajima, T. L. Sun, T. Suekama, Z. L. Wu, S. M. Liang, and J. P. Gong, Macromolecules 45, 9445 (2012).

[28] D. E. Fullenkamp, L. He, D. G. Barrett, W. R. Burghardt, and P. B. Messersmith, Macromolecules 46, 1167 (2013).

[29] S. H. Ku, J. S. Lee, and C. B. Park, Langmuir 26, 15104 (2010).

[30] M.-H. Ryou, Y. M. Lee, J.-K. Park, and J. W. Choi, Adv. Mater. 233066 (2011).

[31] S. Lin, C.-T. Chen, I. Bdikin, V. Ball, J. Gracio, and M. J. Buehler, Soft Matter 10, 457 (2014).

[32] S. M. Kang, S. Park, D. Kim, S. Y. Park, R. S. Ruoff, and H. Lee, Adv. Funct. Mater. 21, 108 (2011).

[33] E. Ducrot, Y. Chen, M. Bulters, R. P. Sijbesma, and C. Creton, Science 344, 186 (2014).

[34] H. Lorenz and M. Klüppel, J. Mech. Phys. Solids 60, 1842 (2012).

[35] A. E. Ehret and M. Itskov, Int. J. Plasticity 25, 901 (2009).

[36] R. Dargazany and M. Itskov, Int. J. Solids Struct. 46, 2967 (2009).

[37] D. Juhre, R. Raghunath, M. Klüppel, and H. Lorenz, in Constitutive Models for Rubber VIII (CRC Press, Boca Raton, FL, 2013), pp. 297-302.

[38] R. Dargazany and M. Itskov, Phys. Rev. E 88, 012602 (2013)

[39] R. Dargazany, V. N. Khiêm, and M. Itskov, Int. J. Plasticity 63, 94 (2014).

[40] V. A. Froltsov, M. Klüppel, and G. Raos, Phys. Rev. E 86, 041801 (2012)

[41] P. Fratzl, J. R. Soc. Interface 4, 637 (2007).

[42] S. Keten and M. J. Buehler, Nano Lett. 8, 743 (2008).

[43] S. Keten, Z. Xu, B. Ihle, and M. J. Buehler, Nat. Mater. 9, 359 (2010).

[44] M. A. Hartmann and P. Fratzl, Nano Lett. 9, 3603 (2009).

[45] A. E. Elbanna and J. M. Carlson, PLOS One 8, e56118 (2013).

[46] C. K. C. Lieou, A. E. Elbanna, and J. M. Carlson, Phys. Rev. E 88, 012703 (2013).

[47] P. Sadhukhan, O. Schumann, and C. Heussinger, Eur. Phys. J. E 37, 58 (2014).

[48] P. Benetatos, A. von der Heydt, and A. Zippelius, New J. Phys. 16, 113037 (2014).

[49] S. S. Nabavi, M. J. Harrington, O. Paris, P. Fratzl, and M. A. Hartmann, New J. Phys. 16, 013003 (2014).

[50] D. Landau and K. Binder, A Guide to Monte Carlo Simulations in Statistical Physics (Cambridge University Press, Cambridge, UK, 2009).

[51] F. Manca, S. Giordano, P. L. Palla, R. Zucca, F. Cleri, and L. Colombo, J. Chem. Phys. 136, 154906 (2012).

[52] J. F. Marko and E. D. Siggia, Macromolecules 28, 8759 (1995).

[53] C. Ortiz and G. Hadziioannou, Macromolecules 32, 780 (1999).

[54] T. Gutsmann, T. Hassenkam, J. A. Cutroni, and P. K. Hansma, Biophys. J. 89, 536 (2005).

[55] J. Kierfeld, T. Kühne, and R. Lipowsky, Phys. Rev. Lett. 95, 038102 (2005).

[56] J. Kierfeld, Phys. Rev. Lett. 97, 058302 (2006).

[57] D. E. Makarov and G. J. Rodin, Phys. Rev. E 66, 011908 (2002).

[58] G. E. Fantner, E. Oroudjev, G. Schitter, L. S. Golde, P. Thurner, M. M. Finch, P. Turner, T. Gutsmann, D. E. Morse, H. Hansma et al., Biophys. J. 90, 1411 (2006).

[59] S. S. Nabavi, M. J. Harrington, P. Fratzl, and M. A. Hartmann, Bioinspired Biomim. Nanobiomater. 3, 139 (2014). 
[60] H. Lee, N. F. Scherer, and P. B. Messersmith, Proc. Natl. Acad Sci. USA 103, 12999 (2006).

[61] M. Rief, M. Gautel, F. Oesterhelt, J. M. Fernandez, and H. E. Gaub, Science 276, 1109 (1997).

[62] A. F. Oberhauser, P. E. Marszalek, H. P. Erickson, and J. M. Fernandez, Nature 393, 181 (1998).

[63] T. E. Fisher, P. E. Marszalek, and J. M. Fernandez, Nat. Struct. Biol. 7, 719 (2000).

[64] T. M. Dugdale, R. Dagastine, A. Chiovitti, P. Mulvaney, and R. Wetherbee, Biophys. J. 89, 4252 (2005).

[65] T. M. Dugdale, R. Dagastine, A. Chiovitti, and R. Wetherbee, Biophys. J. 90, 2987 (2006).

[66] M. J. Harrington and J. H. Waite, in Fibrous Proteins (Landes Bioscience, Austin, TX, 2008), p. 30.

[67] S. L. Brazee and E. Carrington, Biol. Bull. 211, 263 (2006).
[68] M. J. Harrington and J. H. Waite, J. Exp. Biol. 210, 4307 (2007).

[69] N. Holten-Andersen, M. J. Harrington, H. Birkedal, B. P. Lee, P. B. Messersmith, K. Y. C. Lee, and J. H. Waite, Proc. Natl. Acad. Sci. USA 108, 2651 (2011).

[70] M. Krogsgaard, M. A. Behrens, J. S. Pedersen, and H. Birkedal, Biomacromolecules 14, 297 (2013).

[71] K. Eom, D. E. Makarov, and G. J. Rodin, Phys. Rev. E 71, 021904 (2005).

[72] S. Krauss, T. H. Metzger, P. Fratzl, and M. J. Harrington, Biomacromolecules 14, 1520 (2013).

[73] D. T. Seaton, S. Schnabel, D. P. Landau, and M. Bachmann, Phys. Rev. Lett. 110, 028103 (2013).

[74] K. Eom, P.-C. Li, D. E. Makarov, and G. J. Rodin, J. Phys. Chem. B 107, 8730 (2003). 